\title{
NOT SO MANY NON-DISJOINT TRANSLATIONS
}

\author{
ANDRZEJ ROSŁANOWSKI AND VYACHESLAV V. RYKOV \\ (Communicated by Heike Mildenberger)
}

\begin{abstract}
We show that, consistently, there is a Borel set which has uncountably many pairwise very non-disjoint translations, but does not allow a perfect set of such translations.
\end{abstract}

\section{INTRODUCTION}

There is some interest in the literature in Borel sets admitting many pairwise disjoint translations. For instance, Balcerzak, Rosłanowski, and Shelah [1] studied the $\sigma$-ideal of subsets of $\omega_{2}$ generated by Borel sets with a perfect set of pairwise disjoint translations. In this article we are interested in a somewhat dual property of Borel sets: many overlapping translations.

If $B \subseteq{ }^{\omega} 2$ is an uncountable Borel set, then it includes a perfect set $P$, and then for $x, y \in P$ we have

$$
\mathbf{0}, x+y \in(P+x) \cap(P+y) .
$$

Consequently, every uncountable Borel subset of ${ }^{\omega_{2}} 2$ has a perfect set of pairwise non-disjoint translations. However, if we demand that the intersections are more substantial, then the problem of many non-disjoint translations becomes more interesting. One should notice that if $x+b_{0}=y+b_{1}$, then also $x+b_{1}=y+b_{0}$, so if $x \neq y$ and $(B+x) \cap(B+y)$ is finite, then $|(B+x) \cap(B+y)|$ must be even.

Here we investigate the first non-trivial case when $(B+x) \cap(B+y)$ has at least four elements. We show that it is consistent with ZFC that there is a $\Sigma_{2}^{0}$ subset $B$ of the Cantor space ${ }^{\omega} 2$ such that

- for some uncountable set $H \subseteq{ }^{\omega} 2,\left|(B+h) \cap\left(B+h^{\prime}\right)\right| \geq 4$ for all $h, h^{\prime} \in H$, but

- for every perfect set $P \subseteq{ }^{\omega} 2$ there are $x, x^{\prime} \in P$ such that

$$
\left|(B+x) \cap\left(B+x^{\prime}\right)\right| \leq 2 .
$$

Our proof follows the spirit of the proof of Shelah [5, Theorem 1.13], but since we cut on generality, our arguments are more straightforward. We fully utilize the algebraic properties of $\left({ }^{\omega} 2,+\right)$, in particular the fact that all elements of ${ }^{\omega} 2$ are self-inverse.

This line of research will be continued in Rosłanowski and Shelah [4, where we will deal with the general case of $\kappa$ many pairwise non-disjoint translations (getting the full parallel of [5, Theorem 1.13]).

Received by the editors November 11, 2017, and, in revised form, November 14, 2017, May 27, 2018, and August 13, 2018.

2010 Mathematics Subject Classification. Primary 03E35; Secondary 03E15, 54H05. 
Notation and terminology. Our notation is rather standard and compatible with that of classical textbooks (like Jech [2]). However, in forcing we keep the convention that a stronger condition is the larger one.

Ordinal numbers will be denoted by the lower case initial letters of the Greek alphabet $\alpha, \beta, \gamma, \delta, \varepsilon$ and $\zeta, \xi$. Natural numbers (finite ordinals) will be called $i, j, k$ and $\ell, n$.

For a forcing notion $\mathbb{P}$, all $\mathbb{P}$-names for objects in the extension via $\mathbb{P}$ will be denoted with a tilde below (e.g., $\underset{\sim}{h}, \underset{\sim}{T}$ ), and $G_{\mathbb{P}}$ will stand for the canonical $\mathbb{P}$-name for the generic filter in $\mathbb{P}$.

For two sequences $\eta, \nu$ we write $\nu \triangleleft \eta$ whenever $\nu$ is a proper initial segment of $\eta$, and $\nu \unlhd \eta$ when either $\nu \triangleleft \eta$ or $\nu=\eta$. A tree is a $\triangleleft$-downward closed set of sequences.

The set of all sequences of length $n$ and with values in $\{0,1\}$ is denoted by ${ }^{n} 2$ and we let ${ }^{\omega>} 2=\bigcup_{n<\omega}{ }^{n} 2$. For $\sigma \in{ }^{\omega>} 2$ let $[\sigma]=\left\{x \in{ }^{\omega} 2: \sigma \triangleleft x\right\}$. The Cantor space $^{\omega} 2$ of all infinite $0-1$ sequences is equipped with the topology generated by sets of the form $[\sigma]$ and the coordinate-wise addition + modulo 2 . Thus $\left({ }^{\omega} 2,+\right)$ is a topological group.

For a tree $T \subseteq{ }^{\omega>} 2$ we set $[T]=\left\{x \in{ }^{\omega} 2:(\forall n<\omega)(x\lceil n \in T)\}\right.$.

For a set $A \subseteq X \times Y$ and $x \in X$ and $y \in Y$ let

$$
A_{x}=\{z \in Y:(x, z) \in A\} \quad \text { and } \quad A^{y}=\{z \in X:(z, y) \in A\} .
$$

\section{Some technicalities}

Definition 2.1. Let $1<\ell<\omega$. A 4 -arrangement in ${ }^{\ell_{2}}$ is a tuple $\langle a, b, c, d\rangle \subseteq{ }_{2}{ }_{2}$ such that $a<_{\operatorname{lex}} b<_{\operatorname{lex}} c<_{\operatorname{lex}} d$ and

$$
\begin{aligned}
& \min \{k<\ell: a(k) \neq c(k)\}=\min \{k<\ell: b(k) \neq c(k)\} \\
= & \min \{k<\ell: a(k) \neq d(k)\}=\min \{k<\ell: b(k) \neq d(k)\} .
\end{aligned}
$$

Lemma 2.2. Let $15<\ell<\omega$. Suppose that $h:\left[{ }^{\ell} 2\right]^{2} \longrightarrow 2$ is a coloring with the property that

$(\circledast)$ if $a, b, c \in{ }^{\ell} 2$ are distinct, then $h(a, b)=1$ or $h(a, c)=1$, or $h(b, c)=1$.

(That is, there is no h-homogenous triangle in color 0 .) Then there is a set $\mathcal{A} \subseteq{ }_{2}$ such that

(1) $|\mathcal{A}| \geq 5$, and $\mathcal{A}$ contains a 4-arrangement, and

(2) $\mathcal{A}$ is h-homogeneous in color 1 , i.e., $h(a, b)=1$ for distinct $a, b \in \mathcal{A}$.

Proof. First, for $a \in{ }^{\ell} 2$ let $Z_{a}=\left\{x \in{ }^{\ell} 2: x \neq a \wedge h(x, a)=0\right\}$. It follows from the assumption $(\circledast)$ that

(*) for each $a$, the set $Z_{a}$ is $h$-homogenous in color 1 .

If for some $a \in{ }^{\ell} 2$ the set $Z_{a}$ satisfies the requirements of (1), then we are done. So suppose that

$(\odot)$ for each $a \in{ }^{\ell} 2$ either $\left|Z_{a}\right| \leq 4$ or $Z_{a}$ contains no 4 -arrangement.

Let $a \in{ }^{\ell} 2$ be the sequence constantly equal to 0 and let $d \in{ }^{\ell} 2$ be the $<_{\text {lex }}$-last element of ${ }^{\ell} 2 \backslash Z_{a}$. It follows from $(\odot)$ that $\left\{x \in{ }^{\ell} 2: x \uparrow(\ell-3) \equiv 1\right\} \backslash Z_{a} \neq \emptyset$, and hence $d(k)=1$ for $k<\ell-3$.

Let $Y=\left\{\sigma \in{ }^{\ell-3} 2: \sigma(0)=0 \wedge \sigma(1)=1\right\}$ and for $\sigma \in Y$ let $X_{\sigma}=\left\{x \in{ }^{\ell} 2\right.$ : $\sigma \triangleleft x\}$. By $\left(\odot\right.$ ), $X_{\sigma} \backslash Z_{a} \neq \emptyset$ (for each $\sigma \in Y$ ), so we may pick $x_{\sigma} \in X_{\sigma}$ such that $h\left(a, x_{\sigma}\right)=1$. Again by $(\odot)$, the set $\left\{x_{\sigma}: \sigma \in Y\right\}$ cannot be contained in 
$Z_{d}$, so we may pick $\sigma^{*} \in Y$ such that $h\left(d, x_{\sigma^{*}}\right)=1$. Set $b=x_{\sigma^{*}}$ and note that $h(a, b)=h(b, d)=1$.

Now we repeat the above procedure "on $d$ 's side" for both $a$ and $b$ and $d$. We let $Y^{\prime}=\left\{\sigma \in \in^{\ell-3} 2: \sigma(0)=1 \wedge \sigma(1)=0\right\}$ and $Y^{\prime \prime}=\left\{\rho \in^{\ell-6} 2: \sigma(0)=1 \wedge \sigma(1)=0\right\}$. For $\sigma \in Y^{\prime}$ consider $X_{\sigma}=\left\{x \in{ }^{\ell} 2: \sigma \triangleleft x\right\}$ and note that by our assumptions we may pick $x_{\sigma}^{\prime} \in X_{\sigma}$ such that $h\left(a, x_{\sigma}^{\prime}\right)=1$. Now, for each $\rho \in Y^{\prime \prime}$ we may choose $\sigma_{\rho} \in Y^{\prime}$ such that $\rho \triangleleft \sigma_{\rho}$ and $h\left(b, x_{\sigma_{\rho}}^{\prime}\right)=1$. By our assumptions, for some $\rho^{*} \in Y^{\prime \prime}$ we also have $h\left(d, x_{\sigma_{\rho^{*}}}^{\prime}\right)=1$. Set $c=x_{\sigma_{\rho^{*}}}^{\prime}$ and note that $\langle a, b, c, d\rangle$ is a 4 -arrangement which is homogenous in color 1.

Repeating the above procedure again, but starting with $Y^{+}=\left\{\sigma \in{ }^{\ell-3} 2: \sigma(0)=\right.$ $\sigma(1)=0 \wedge \sigma(2)=1\}$, going through levels $\ell-3, \ell-6$, and $\ell-9$ and dealing with $a, b, c, d$ one may find $e \in{ }^{\ell} 2$ such that $\mathcal{A}=\{a, b, c, d, e\}$ satisfies the demands $(1)+(2)$.

Lemma 2.3. Let $0<\ell<\omega$ and let $\mathcal{B} \subseteq{ }^{\ell} 2$ be a linearly independent set of vectors $\left(\right.$ in $\left({ }^{\ell} 2,+, \cdot\right)$ over $\left.\left(2,+_{2}, \cdot 2\right)\right)$.

(a) If $a, b, c \in{ }^{\ell} 2$ are pairwise distinct and $\{a, b, c\}+\{a, b, c\} \subseteq \mathcal{B}+\mathcal{B}$, then for some pairwise distinct $\eta, \nu, \rho \in \mathcal{B}$ we have

$$
a+b=\eta+\nu \quad \text { and } \quad a+c=\eta+\rho .
$$

(b) If $\mathcal{A} \subseteq{ }^{\ell} 2,|\mathcal{A}| \geq 5$, and $\mathcal{A}+\mathcal{A} \subseteq \mathcal{B}+\mathcal{B}$, then for a unique $x \in{ }^{\ell}$ we have $\mathcal{A}+x \subseteq \mathcal{B}$.

Proof. (a) Let $\nu_{a}, \nu_{b}, \eta_{a}, \eta_{c}, \rho_{b}, \rho_{c} \in \mathcal{B}$ be such that

$$
a+b=\nu_{a}+\nu_{b}, \quad a+c=\eta_{a}+\eta_{c}, \quad \text { and } \quad b+c=\rho_{b}+\rho_{c} .
$$

Then $\nu_{a} \neq \nu_{b}, \eta_{a} \neq \eta_{c}, \rho_{b} \neq \rho_{c}$, and

$$
\rho_{b}+\rho_{c}=b+c=a+b+a+c=\nu_{a}+\nu_{b}+\eta_{a}+\eta_{c} .
$$

By the linear independence of $\mathcal{B}$ we conclude $\left\{\nu_{a}, \nu_{b}\right\} \cap\left\{\eta_{a}, \eta_{c}\right\} \neq \emptyset$.

(b) Let $\mathcal{A}=\left\{a_{0}, a_{1}, \ldots, a_{n-1}\right\}, n=|\mathcal{A}| \geq 5$. Using clause (a) we may choose $\eta, \nu, \rho \in \mathcal{B}$ such that

$$
a_{0}+a_{1}=\eta+\nu \quad \text { and } \quad a_{0}+a_{2}=\eta+\rho .
$$

Note that

$(\oplus)$ if $2<k<n$ and $a_{0}+a_{k}=\eta^{*}+\eta^{+}$with $\eta^{*}, \eta^{+} \in \mathcal{B}$, then

$$
\left[\left\{\eta^{*}, \eta^{+}\right\} \cap\{\eta, \nu\}|=|\left\{\eta^{*}, \eta^{+}\right\} \cap\{\eta, \rho\} \mid=1 .\right.
$$

(Just apply clause (a) to $a_{0}, a_{1}, a_{k}$ and to $a_{0}, a_{2}, a_{k}$, remembering that $\mathcal{B}$ is linearly independent.)

Let $x=a_{0}+\eta$. We will argue that $a_{i}+x \in \mathcal{B}$ for all $i<n$. Clearly by our choices this holds for $i \leq 2$. Suppose $2<i<n$ is such that $a_{i}+x \notin \mathcal{B}$. Let $\eta^{*}, \eta^{+} \in \mathcal{B}$ be such that $a_{0}+a_{i}=\eta^{*}+\eta^{+}$. If we had $\eta \in\left\{\eta^{*}, \eta^{+}\right\}$, then $a_{i}+\left(a_{0}+\eta\right) \in \mathcal{B}$, contradicting $a_{i}+x \notin \mathcal{B}$. Therefore $\eta \notin\left\{\eta^{*}, \eta^{+}\right\}$and it follows from $(\oplus)$ that $\nu, \rho \in\left\{\eta^{*}, \eta^{+}\right\}$, so $a_{0}+a_{i}=\nu+\rho$. Let $j<n$ be such that $j \notin\{0,1,2, i\}$, and let $a_{0}+a_{j}=\nu^{*}+\nu^{+}, \nu^{*}, \nu^{+} \in \mathcal{B}$. Then (using $(\oplus)$ ) we get

$$
\left|\left\{\nu^{*}, \nu^{+}\right\} \cap\{\eta, \nu\}\right|=\left|\left\{\nu^{*}, \nu^{+}\right\} \cap\{\eta, \rho\}\right|=\left|\left\{\nu^{*}, \nu^{+}\right\} \cap\{\nu, \rho\}\right|=1,
$$

a contradiction. The uniqueness of $x$ follows from the linear independence of $\mathcal{B}$. 
Lemma 2.4. Suppose that $P \subseteq \omega_{2}$ is a perfect set and $A_{n} \subseteq P \times P$ are $\Sigma_{1}^{1}$ sets (for $n<\omega)$ such that $P \times P=\bigcup_{n<\omega} A_{n} \cup\{(x, x): x \in P\}$. Then there is a perfect set $P^{*} \subseteq P$ with the following property:

$(\mathrm{D})$ For some increasing sequence of integers $0=n_{0}<n_{1}<n_{2}<n_{3}<\ldots$, for each $k<\omega$ and any distinct $x, y \in P^{*}$ we have

(a) if $x\left\lceil n_{k+1} \neq y\left\lceil n_{k+1}\right.\right.$, then for all $x^{\prime}, y^{\prime} \in P^{*}$

$$
\left(x \left\lceiln_{k+1}=x^{\prime}\left\lceiln _ { k + 1 } \wedge y \left\lceil n_{k+1}=y^{\prime}\left\lceil n_{k+1}\right) \Rightarrow\left((x, y) \in A_{k} \Leftrightarrow\left(x^{\prime}, y^{\prime}\right) \in A_{k}\right)\right.\right. \text {, }\right.\right.
$$

(b) the set $\left\{z\left\lceil n_{k+1}: z \in P^{*} \wedge z\left\lceil n_{k}=x\left\lceil n_{k}\right\}\right.\right.\right.$ has exactly two elements.

Proof. We will use the general result of Mycielski on the existence of independent sets in topological algebras. To be able to quote his theorem we have to introduce some definitions.

We say that a set $S \subseteq P^{m}$ is obtained by identification of variables from $R \subseteq$ $P^{m+1}$ if for some $i, j \leq m$ we have

$$
\left(x_{1}, \ldots, x_{m}\right) \in S \Leftrightarrow\left(x_{1}, \ldots, x_{i}, x_{j}, x_{i+1}, \ldots, x_{m}\right) \in R .
$$

For $n<\omega$ let $J_{n}$ consist of all pairs $(\sigma, \rho) \in{ }^{\omega>} 2 \times{ }^{\omega>}>2$ such that the set $([\sigma] \times[\rho]) \cap A_{n}$ is meager (in $P^{2}$ ) and $[\sigma] \cap[\rho]=\emptyset$, and let $K_{n}$ consist of pairs $(\sigma, \rho) \in{ }^{\omega>} 2 \times{ }^{\omega>} 2$ for which $([\sigma] \times[\rho]) \backslash A_{n}$ is meager and $[\sigma] \cap[\rho]=\emptyset$. For $(\sigma, \rho) \in J_{n}$ we may fix a Borel meager (in $P$ ) set $X_{\sigma, \rho} \subseteq P$ such that

$\left(\forall x \in[\sigma] \backslash X_{\sigma, \rho}\right)\left(\left(A_{n}\right)_{x} \cap[\rho]\right.$ is meager $)$ and $\left(\forall y \in[\rho] \backslash X_{\sigma, \rho}\right)\left(\left(A_{n}\right)^{y} \cap[\sigma]\right.$ is meager $)$.

Similarly, if $(\sigma, \rho) \in K_{n}$, then a Borel meager set $X_{\sigma, \rho} \subseteq P$ is such that

$\left(\forall x \in[\sigma] \backslash X_{\sigma, \rho}\right)\left([\rho] \backslash\left(A_{n}\right)_{x}\right.$ is meager $)$ and

$\left(\forall y \in[\rho] \backslash X_{\sigma, \rho}\right)\left([\sigma] \backslash\left(A_{n}\right)^{y}\right.$ is meager $)$.

For $(\sigma, \rho) \in J_{n} \cup K_{n}$ let

$$
\begin{aligned}
& R_{\sigma, \rho}^{n}=\left\{\left(x_{1}, x_{2}, y_{1}, y_{2}\right) \in P^{4}:\right. x_{1}, x_{2} \in[\sigma] \backslash X_{\sigma, \rho} \wedge y_{1}, y_{2} \in[\rho] \backslash X_{\sigma, \rho} \\
&\left.\wedge\left(x_{1}, y_{1}\right) \in A_{n} \wedge\left(x_{2}, y_{2}\right) \notin A_{n}\right\} .
\end{aligned}
$$

Clearly for every $(\sigma, \rho) \in J_{n} \cup K_{n}$ the set $R_{\sigma, \rho}^{n}$ is meager (in $P^{4}$ ), moreover if $S \subseteq P^{k}$ is obtained from $R_{\sigma, \rho}^{n}$ by repeated identification and/or permutation of variables, then $S$ is meager in $P^{k}$ as well.

The sets $A_{n}$ have the Baire property and hence the sets $J_{n} \cup K_{n}$ are dense in ${ }^{\omega>} 2 \times{ }^{\omega>} 2$. Let

$$
X=\bigcup\left\{X_{\sigma, \rho}:(\sigma, \rho) \in J_{n} \cup K_{n} \wedge n<\omega\right\}
$$

and

$R_{n}=\left\{(x, y) \in P^{2}: x \neq y\right.$ and for all $(\sigma, \rho) \in J_{n} \cup K_{n}$ we have $\left.x \notin[\sigma] \vee y \notin[\rho]\right\}$.

Easily, $X$ is a meager subset of $P$, each $R_{n}$ is meager in $P^{2}$ and identification of variables in $R_{n}$ leads to an empty set (so meager).

By [3, Theorem 1, p. 141] there is a perfect set $P^{\prime} \subseteq P$ such that

- $\left(P^{\prime} \times P^{\prime} \times P^{\prime} \times P^{\prime}\right) \cap R_{\sigma, \rho}^{n}=\emptyset$ for all $n<\omega$ and $(\sigma, \rho) \in J_{n} \cup K_{n}$, and

- $P^{\prime} \cap X=\emptyset$ and $\left(P^{\prime} \times P^{\prime}\right) \cap R_{n}=\emptyset$ for all $n<\omega$. 
Clearly, if $x \neq y$ are from $P^{\prime}$ and $n<\omega$, then for some $N<\omega$ we have

$$
\left(\forall x^{\prime}, y^{\prime} \in P^{\prime}\right)\left(\left(x \left\lceilN=x^{\prime} \uparrow N \wedge y\left\lceil N=y^{\prime}\lceil N) \Rightarrow\left((x, y) \in A_{n} \Leftrightarrow\left(x^{\prime}, y^{\prime}\right) \in A_{n}\right)\right) .\right.\right.\right.
$$

By shrinking the perfect $P^{\prime}$ one can construct a perfect set $P^{*} \subseteq P^{\prime}$ and an increasing sequence $0=n_{0}<n_{1}<n_{2}<\ldots$ such that the demands in $(\mathcal{Q})$ are satisfied.

\section{The MAIn RESUlt}

Theorem 3.1. There exists a ccc forcing notion $\mathbb{P}$ of size $\omega_{1}$ such that in $\mathbf{V}^{\mathbb{P}}$, there is a $\Sigma_{2}^{0}$ set $B \subseteq{ }^{\omega} 2$ with the properties that

(\$) (a) for some sequence $\left\langle h_{\alpha}: \alpha<\omega_{1}\right\rangle$ of pairwise distinct elements of ${ }^{\omega} 2$ we have $\left|\left(h_{\alpha}+B\right) \cap\left(h_{\beta}+B\right)\right| \geq 4$, but

(b) in each perfect set $P \subseteq{ }^{\omega} 2$ there are $f, g \in P$ with $|(f+B) \cap(g+B)| \leq 2$.

Proof. A condition $p \in \mathbb{P}$ is a tuple

$$
p=\left\langle u, n, \bar{\eta}, m_{*}, \bar{t}, \mu, K\right\rangle=\left\langle u^{p}, n^{p}, \bar{\eta}^{p}, m_{*}^{p}, \bar{t}^{p}, \mu^{p}, K^{p}\right\rangle
$$

satisfying the following demands:

(1) $\emptyset \neq u \in\left[\omega_{1}\right]^{<\omega}, 0<m_{*}, n<\omega$, and $\bar{\eta}=\left\langle\eta_{\alpha}: \alpha \in u\right\rangle \subseteq{ }^{n} 2$.

(2) $\bar{t}=\left\langle t_{m}: m<m_{*}\right\rangle$, each $t_{m} \subseteq n \geq_{2}$ is a tree with all maximal nodes of length $n$.

(3) $\mu:[u]^{2} \longrightarrow{ }^{n} 2 \times m_{*}$, and if $\alpha \neq \beta$ are from $u$, then we will write $\mu(\alpha, \beta)=$ $\mu(\beta, \alpha)=\left(\rho_{\alpha, \beta}, \ell_{\alpha, \beta}\right)$.

(4) If $\alpha \neq \beta$ are from $u$, then both $\eta_{\alpha}+\rho_{\alpha, \beta} \in t_{\ell_{\alpha, \beta}}$ and $\eta_{\beta}+\rho_{\alpha, \beta} \in t_{\ell_{\alpha, \beta}}$.

(5) $K: u \longrightarrow m_{*}: \alpha \mapsto K_{\alpha}$ and $\eta_{\alpha} \in t_{K_{\alpha}}$.

(6) If $\alpha<\beta<\gamma$ are from $u$, then $\left\{K_{\alpha}, K_{\gamma}, \ell_{\alpha, \gamma}\right\} \neq\left\{K_{\beta}, K_{\gamma}, \ell_{\beta, \gamma}\right\}$.

(7) If $m<m^{\prime}<m_{*}$, then $t_{m} \cap t_{m^{\prime}} \cap{ }^{n} 2=\emptyset$.

(8) If $m<m_{*}$, then $t_{m} \cap{ }^{n} 2 \subseteq\left\{\eta_{\alpha}+\rho_{\alpha, \beta}: \alpha \neq \beta \wedge \alpha, \beta \in u\right\} \cup\left\{\eta_{\alpha}: \alpha \in u\right\}$.

(9) $\left\langle\eta_{\alpha}: \alpha \in u\right\rangle \frown\left\langle\rho_{\alpha, \beta}: \alpha<\beta \wedge \alpha, \beta \in u\right\rangle$ is a list of linearly independent vectors (in $\left({ }^{n} 2,+, \cdot\right)$ over $\left.\left(2,+_{2}, \cdot 2\right)\right)$; in particular they are pairwise distinct.

The order $\leq_{\mathbb{P}}=\leq$ of $\mathbb{P}$ is defined by:

$p \leq q$ if and only if the following conditions are satisfied:

(i) $u^{p} \subseteq u^{q}, n^{p} \leq n^{q}$, and $m_{*}^{p} \leq m_{*}^{q}$.

(ii) If $\alpha \in u^{p}$, then $\eta_{\alpha}^{q}\left\lceil n^{p}=\eta_{\alpha}^{p}\right.$.

(iii) If $m<m_{*}^{p}$, then $t_{m}^{q} \cap n^{p} 2=t_{m}^{p} \cap n^{n^{p}} 2$.

(iv) If $\alpha \in u^{p}$, then $K_{\alpha}^{p}=K_{\alpha}^{q}$ and if $\alpha \neq \beta$ are from $u^{p}$, then $\ell_{\alpha, \beta}^{p}=\ell_{\alpha, \beta}^{q}$ and $\rho_{\alpha, \beta}^{p} \unlhd \rho_{\alpha, \beta}^{q}$.

Claim 3.1.1. ( $\mathbb{P}, \leq)$ is a partial order of size $\omega_{1}$.

Claim 3.1.2.

(1) If $p \in \mathbb{P}$ and $b_{0}, c_{0}, b_{1}, c_{1} \in \bigcup_{m<m_{*}^{p}}\left(t_{m}^{p} \cap n^{p} 2\right)$ are pairwise distinct and satisfy $b_{0}+c_{0}=b_{1}+c_{1}$, then for some $\alpha \neq \beta$ from $u^{p}$ we have

$$
\left\{b_{0}, c_{0}, b_{1}, c_{1}\right\}=\left\{\eta_{\alpha}^{p}, \eta_{\beta}^{p}, \eta_{\alpha}^{p}+\rho_{\alpha, \beta}^{p}, \eta_{\beta}^{p}+\rho_{\alpha, \beta}^{p}\right\} .
$$

(2) Also, for some $i<2,\left\{b_{i}, c_{i}\right\}=\left\{\eta_{\alpha}^{p}, \eta_{\beta}^{p}\right\}$ or $\left\{b_{i}, c_{i}\right\}=\left\{\eta_{\alpha}^{p}+\rho_{\alpha, \beta}^{p}, \eta_{\beta}^{p}\right\}$ or $\left\{b_{i}, c_{i}\right\}=\left\{\eta_{\alpha}^{p}+\rho_{\alpha, \beta}^{p}, \eta_{\alpha}^{p}\right\}$. 
Proof of the claim. It follows from the definition of $\mathbb{P}$ (clause (8)) that $b_{0}, c_{0}, b_{1}, c_{1}$ $\in\left\{\eta_{\alpha}, \eta_{\alpha}+\rho_{\alpha, \beta}: \alpha \neq \beta\right.$ are from $\left.u^{p}\right\}$. Since, by clause (9), $\left\langle\eta_{\alpha}^{p}: \alpha \in u\right\rangle \frown\left\langle\rho_{\alpha, \beta}\right.$ : $\alpha<\beta \wedge \alpha, \beta \in u\rangle$ are linearly independent we easily get our conclusion.

Claim 3.1.3. For every $N, M<\omega$ and $\delta<\omega_{1}$ the set

$$
Z_{\delta}^{N, M}=\left\{p \in \mathbb{P}: n^{p} \geq N \wedge m_{*}^{p} \geq M \wedge \delta \in u^{p}\right\}
$$

is open dense in $\mathbb{P}$.

Proof of the claim. Suppose that $p \in \mathbb{P}$ and let $\alpha \in \omega_{1} \backslash u^{p}$.

Let $\left\langle\alpha_{0}, \ldots, \alpha_{k}\right\rangle$ be the increasing enumeration of $u^{p}$. Set $u=u^{p} \cup\{\alpha\}, n=$ $n^{p}+k+2$, and $m_{*}=m_{*}^{p}+k+2$. For $i \leq k$ let

$$
\eta_{\alpha_{i}}=\eta_{\alpha_{i}}^{p} \frown\langle\underbrace{0, \ldots, 0}_{k+2}\rangle \text { and } \rho_{\alpha_{i}, \alpha}=\rho_{\alpha, \alpha_{i}}=\langle\underbrace{0, \ldots, 0}_{n^{p}+i+1}\rangle\langle 1\rangle \curlyvee\langle\underbrace{0, \ldots, 0}_{k-i}\rangle \text {. }
$$

We also let $\eta_{\alpha}=\langle\underbrace{0, \ldots, 0}_{n^{p}}\rangle\langle 1\rangle \curlyvee\langle\underbrace{0, \ldots, 0}_{k+1}\rangle$ and we put $\ell_{\alpha_{i}, \alpha}=\ell_{\alpha, \alpha_{i}}=m_{*}^{p}+i$ and $K_{\alpha}=m_{*}^{p}+k+1$. Next, for $i \leq k$ we define $K_{\alpha_{i}}=K_{\alpha_{i}}^{p}$ and for $i<j \leq k$ we let $\rho_{\alpha_{j}, \alpha_{i}}=\rho_{\alpha_{i}, \alpha_{j}}=\rho_{\alpha_{i}, \alpha_{j}}^{p}\langle\underbrace{0, \ldots, 0}_{k+2}\rangle$, and $\ell_{\alpha_{j}, \alpha_{i}}=\ell_{\alpha_{i}, \alpha_{j}}=\ell_{\alpha_{i}, \alpha_{j}}^{p}$. (So a function $\mu:[u]^{2} \longrightarrow{ }^{n} 2 \times m_{*}$ is defined now too.) For $m<m_{*}^{p}$ let

$$
t_{m}=t_{m}^{p} \cup\left\{\sigma \curlyvee\langle\underbrace{0, \ldots, 0}_{j}\rangle: \sigma \in t_{m}^{p} \cap n^{n^{p}} 2 \wedge j<k+3\right\}
$$

and for $m=m_{*}^{p}+i<m_{*}-1$ let $t_{m}=\left\{\left(\eta_{\alpha_{i}}+\rho_{\alpha_{i}, \alpha}\right)\left\lceil j,\left(\eta_{\alpha}+\rho_{\alpha_{i}, \alpha}\right)\lceil j: j \leq n\}\right.\right.$ and $t_{m_{*}-1}=\left\{\eta_{\alpha}\lceil j: j \leq n\}\right.$. Finally, let $\bar{t}=\left\langle t_{m}: m<m_{*}\right\rangle$.

It is straightforward to verify that $q=\left\langle u, n, \bar{\eta}, m_{*}, \bar{t}, \mu, K\right\rangle$ satisfies the demands of the definition of a condition in $\mathbb{P}$. Moreover, $q$ is a condition stronger than $p$, and $\alpha \in u^{q}, m_{*}^{q} \geq m_{*}^{p}+2$ and $n^{q} \geq n^{p}+2$.

Now the claim readily follows.

Claim 3.1.4. The forcing notion $\mathbb{P}$ has the Knaster property.

Proof of the claim. Suppose that $\left\langle p_{\xi}: \xi<\omega_{1}\right\rangle$ is a sequence of conditions from $\mathbb{P}$. Applying the standard $\Delta$-lemma based cleaning procedure we may find an uncountable set $A \subseteq \omega_{1}$ such that $\left\{u^{p_{\xi}}: \xi \in A\right\}$ forms a $\Delta$-system of finite sets and for $\xi<\zeta$ from $A$ we have:

$(*)_{1} n^{p_{\xi}}=n^{p_{\zeta}}, m_{*}^{p_{\xi}}=m_{*}^{p_{\zeta}}, \bar{t}^{p_{\xi}}=\bar{t}^{p_{\zeta}}$,

$(*)_{2}\left|u^{p_{\xi}}\right|=\left|u^{p_{\zeta}}\right|, u^{p_{\xi}} \cap u^{p_{\zeta}}$ is an initial segment of both $u^{p_{\xi}}$ and $u^{p_{\zeta}}$ and $\max \left(u^{p_{\xi}} \backslash u^{p_{\zeta}}\right)<\min \left(u^{p_{\zeta}} \backslash u^{p_{\xi}}\right)$,

$(*)_{3}$ if $\pi: u^{p_{\xi}} \longrightarrow u^{p_{\zeta}}$ is the order preserving bijection, then for every $\alpha \in u^{p_{\xi}}$ we have

$$
K_{\alpha}^{p_{\xi}}=K_{\pi(\alpha)}^{p_{\zeta}} \quad \text { and } \quad \eta_{\alpha}^{p_{\xi}}=\eta_{\pi(\alpha)}^{p_{\zeta}}
$$

and $\mu^{p_{\xi}}(\alpha, \beta)=\mu^{p_{\zeta}}(\pi(\alpha), \pi(\beta))$ for all $\alpha<\beta$ from $u^{p_{\xi}}$.

We may assume that $u^{p_{\xi}} \cap u^{p_{\zeta}} \neq \emptyset \neq u^{p_{\xi}} \backslash u^{p_{\zeta}}$ for distinct $\xi, \zeta \in A$.

We will argue that if $\xi<\zeta$ are from $A$, then the conditions $p_{\xi}, p_{\zeta}$ are compatible. 
Let

$(*)_{4}\left\langle\gamma_{0}, \ldots, \gamma_{k_{0}}\right\rangle$ be the increasing enumeration of $u^{p_{\xi}} \cap u^{p_{\zeta}}$, let $\left\langle\alpha_{0}, \ldots, \alpha_{k_{1}}\right\rangle$ be the increasing enumeration of $u^{p_{\xi}} \backslash u^{p_{\zeta}}$, and let $\left\langle\beta_{0}, \ldots, \beta_{k_{1}}\right\rangle$ be the increasing enumeration of $u^{p_{\zeta}} \backslash u^{p_{\xi}}$ (so $\left.2+k_{0}+k_{1}=\left|u^{p_{\xi}}\right|\right)$ and let

$(*)_{5} k^{*}=\left(k_{1}+1\right)\left(k_{0}+k_{1}+3\right)+\frac{\left(k_{1}-1\right)\left(k_{1}+2\right)}{2}+1, n=n^{p_{\xi}}+k^{*}$, and $m_{*}=$ $m_{*}^{p_{\xi}}+\left(k_{1}+1\right)^{2}$, and

$(*)_{6} \nu_{i}=\langle\underbrace{0, \ldots, 0}_{i}\rangle \frown\langle 1\rangle \frown\langle\underbrace{0, \ldots, 0}_{k^{*}-i-1}\rangle \in k^{*} 2$ for $i<k^{*}$.

To define a common upper bound to $p_{\xi}$ and $p_{\zeta}$ we put

$(*)_{7} u=u^{p_{\xi}} \cup u^{p_{\zeta}}=\left\{\alpha_{i}: i \leq k_{1}\right\} \cup\left\{\beta_{i}: i \leq k_{1}\right\} \cup\left\{\gamma_{i}: i \leq k_{0}\right\}$, and

$(*)_{8}$ for $i \leq k_{1}$ we set $\eta_{\alpha_{i}}=\eta_{\alpha_{i}}^{p_{\xi}} \smile\langle\underbrace{0, \ldots, 0}_{n-n^{p_{\xi}}}\rangle, \eta_{\beta_{i}}=\eta_{\beta_{i}}^{p_{\zeta}} \nu_{i}$ and for $i \leq k_{0}$ we let $\eta_{\gamma_{i}}=\eta_{\gamma_{i}}^{p_{\xi}} \frown\langle\underbrace{0, \ldots, 0}_{n-n^{p_{\xi}}}\rangle$, and

$(*)_{9} K_{\alpha_{i}}=K_{\alpha_{i}}^{p_{\xi}}, K_{\beta_{i}}=K_{\beta_{i}}^{p_{\zeta}}\left(\right.$ for $\left.i \leq k_{1}\right)$ and $K_{\gamma_{i}}=K_{\gamma_{i}}^{p_{\xi}}$ (for $\left.i \leq k_{0}\right)$.

We define also a function $\mu:[u]^{2} \longrightarrow{ }^{n} 2 \times m_{*}:\{\delta, \varepsilon\} \mapsto\left(\rho_{\delta, \varepsilon}, \ell_{\delta, \varepsilon}\right)$ as follows:

$(*)_{10}$ if $\delta<\varepsilon$ are from $u^{p_{\xi}}$, then $\rho_{\delta, \varepsilon}=\rho_{\delta, \varepsilon}^{p_{\xi}} \frown\langle\underbrace{0, \ldots, 0}_{n-n^{p_{\xi}}}\rangle$ and $\ell_{\delta, \varepsilon}=\ell_{\delta, \varepsilon}^{p_{\xi}}$;

$(*)_{11}$ if $i \leq k_{0}$ and $j \leq k_{1}$, then $\rho_{\gamma_{i}, \beta_{j}}=\rho_{\gamma_{i}, \beta_{j}}^{p_{\zeta}} \frown_{k}$, where $k=\left(k_{1}+1\right)+i\left(k_{1}+\right.$ 1) $+j$, and $\ell_{\gamma_{i}, \beta_{j}}=\ell_{\gamma_{i}, \beta_{j}}^{p_{\zeta}}$;

$(*)_{12}$ if $i, j \leq k_{1}$, then $\rho_{\alpha_{i}, \beta_{j}}=\langle\underbrace{0, \ldots, 0}_{n^{p_{\zeta}}}\rangle \nu_{k}$, where $k=\left(k_{0}+2\right)\left(k_{1}+1\right)+$ $i\left(k_{1}+1\right)+j$, and $\ell_{\alpha_{i}, \beta_{j}}=m_{*}^{p_{\zeta}}+i\left(k_{1}+1\right)+j ;$

$(*)_{13}$ if $i<j \leq k_{1}$, then $\rho_{\beta_{i}, \beta_{j}}=\rho_{\beta_{i}, \beta_{j}}^{p_{\zeta}} \frown \nu_{k}$, where $k=\left(k_{1}+1\right)\left(k_{0}+k_{1}+3\right)+$ $\frac{i\left(2 k_{1}-i+1\right)}{2}+(j-i-1)$, and $\ell_{\beta_{i}, \beta_{j}}=\ell_{\beta_{i}, \beta_{j}}^{p_{\zeta}}$.

Finally, let $\bar{t}=\left\langle t_{m}: m<m_{*}\right\rangle$, where

$(*)_{14}$ if $m<m_{*}^{p_{\zeta}}$, then

$$
\begin{aligned}
t_{m}= & t_{m}^{p_{\zeta}} \cup\left\{\sigma \frown\langle\underbrace{0, \ldots, 0}_{k}\rangle: \sigma \in t_{m}^{p_{\zeta}} \cap n^{n^{p_{\zeta}}} 2 \wedge k \leq k^{*}\right\} \\
& \cup\left\{\eta_{\beta_{i}}\left\lceil k: i \leq k_{1} \wedge K_{\beta_{i}}=m \wedge k \leq n\right\}\right. \\
& \cup\left\{\left(\eta_{\gamma_{i}}+\rho_{\gamma_{i}, \beta_{j}}\right)\left\lceil k: i \leq k_{0} \wedge j \leq k_{1} \wedge \ell_{\gamma_{i}, \beta_{j}}=m \wedge k \leq n\right\}\right. \\
& \cup\left\{\left(\eta_{\beta_{j}}+\rho_{\gamma_{i}, \beta_{j}}\right)\left\lceil k: i \leq k_{0} \wedge j \leq k_{1} \wedge \ell_{\gamma_{i}, \beta_{j}}=m \wedge k \leq n\right\}\right. \\
& \cup\left\{\left(\eta_{\beta_{i}}+\rho_{\beta_{i}, \beta_{j}}\right)\left\lceil k: i<j \leq k_{1} \wedge \ell_{\beta_{i}, \beta_{j}}=m \wedge k \leq n\right\}\right. \\
& \cup\left\{\left(\eta_{\beta_{j}}+\rho_{\beta_{i}, \beta_{j}}\right)\left\lceil k: i<j \leq k_{1} \wedge \ell_{\beta_{i}, \beta_{j}}=m \wedge k \leq n\right\}\right.
\end{aligned}
$$

and

$(*)_{15}$ for $m=m_{*}^{p_{\zeta}}+i\left(k_{1}+1\right)+j<m_{*}, i, j \leq k_{1}$, we let

$$
t_{m}=\left\{( \eta _ { \alpha _ { i } } + \rho _ { \alpha _ { i } , \beta _ { j } } ) \left\lceilk,\left(\eta_{\beta_{j}}+\rho_{\alpha_{i}, \beta_{j}}\right)\lceil k: k \leq n\} .\right.\right.
$$

One easily verifies that $q=\left\langle u, n, \bar{\eta}, m_{*}, \bar{t}, \mu, K\right\rangle$ satisfies the demands of the definition of a condition in $\mathbb{P}$ and that this condition is a common upper bound of $p_{\zeta}$ and $p_{\xi}$. 
We define $\mathbb{P}$-names $\underset{\sim}{h}\left(\right.$ for $\left.\alpha<\omega_{1}\right), \underset{\sim}{T}$ (for $\left.m<\omega\right)$ and $\underset{\sim}{\underset{\gamma}{\alpha}, \beta}\left(\right.$ for $\left.\alpha<\beta<\omega_{1}\right)$ by

- $\Vdash_{\mathbb{P}} " \underset{\sim}{h}=\bigcup\left\{\eta_{\alpha}^{p}: p \in G_{\mathbb{P}} \wedge \alpha \in u^{p}\right\} "$,

- $\vdash_{\mathbb{P}} " \underset{\sim}{T} T_{m}=\bigcup\left\{t_{m}^{p}: p \in G_{\mathbb{P}} \wedge m<m_{*}^{p}\right\} "$,

- $\vdash_{\mathbb{P}} "{\underset{\sim}{\alpha} \alpha, \beta}_{\alpha}=\bigcup\left\{\rho_{\alpha, \beta}^{p}: p \in G_{\mathbb{P}} \wedge \alpha, \beta \in u^{p}\right\} "$.

Claim 3.1.5. For $\alpha<\beta<\omega_{1}$ and $m<\omega$ we have

(1) $\Vdash_{\mathbb{P}}$ “ $\underset{\sim}{\operatorname{h}}, \underset{\sim}{r} \underset{\alpha, \beta}{ } \in{ }^{\omega} 2$ ",

(2) $\Vdash_{\mathbb{P}}$ " $\underset{\sim}{T} T_{m} \subseteq \omega>2$ is a tree with no maximal nodes ",

(3) $\Vdash_{\mathbb{P}}$ " if $m<m^{\prime}<\omega$, then $[\underset{\sim}{T} m] \cap\left[\underset{\sim}{T} m^{\prime}\right]=\emptyset "$.

Proof of the claim. By Claim 3.1 .3 and the definition of the order of $\mathbb{P}$.

Let $\underset{\sim}{B}$ be the $\mathbb{P}$-name for the $\Sigma_{2}^{0}$ subset of ${ }^{\omega} 2$ given by $\Vdash_{\mathbb{P}}$ " $\underset{\sim}{B}=\bigcup_{m<\omega}[\underset{\sim}{T} m]$ ".

Claim 3.1.6. For each $\alpha<\beta<\omega_{1}$ we have

$$
\Vdash_{\mathbb{P}} "\left|(\underset{\sim}{(h}+\underset{\sim}{B}) \cap\left(\underset{\sim}{h}{ }_{\beta}+\underset{\sim}{B}\right)\right| \geq 4 " .
$$

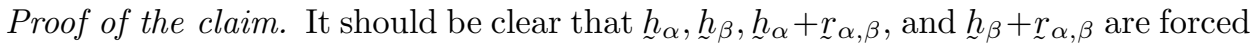
to belong to $\underset{\sim}{B}$ and they all are pairwise distinct. Therefore, $\mathbf{0}, \underset{\sim}{r_{\alpha}, \beta}, \underset{\sim}{h}{ }_{\alpha}+\underset{\sim}{\operatorname{h}} \underset{\beta}{ }$ and

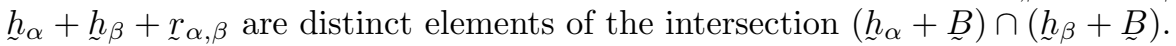

Claim 3.1.7.

$\Vdash_{\mathbb{P}}$ " for every perfect set $P \subseteq{ }^{\omega} 2$ there are $f, g \in P$ with $|(f+\underset{\sim}{B}) \cap(g+\underset{\sim}{B})|<4$ ".

Proof of the claim. Suppose $G \subseteq \mathbb{P}$ is generic over $\mathbf{V}$ and let us work in $\mathbf{V}[G]$ for awhile. Assume towards contradiction that $P \subseteq{ }^{\omega} 2$ is a perfect set such that

$$
(\forall f, g \in P)\left(\left|\left(f+{\underset{\sim}{B}}^{G}\right) \cap\left(g+{\underset{\sim}{B}}^{G}\right)\right| \geq 4 .\right.
$$

Then for distinct $f, g \in P$ there are pairwise distinct $b_{0}, c_{0}, b_{1}, c_{1} \in B$ such that $f+g=b_{0}+c_{0}=b_{1}+c_{1}$. Now, for $\left(\ell_{0}, m_{0}, \ell_{1}, m_{1}, N\right) \in{ }^{5} \omega$ let

$$
\begin{array}{r}
A_{\ell_{0}, m_{0}, \ell_{1}, m_{1}}^{N}=\left\{(f, g) \in P^{2}: \text { for some } b_{i} \in\left[\underset{\sim}{T_{\ell_{i}}^{G}}\right], c_{i} \in\left[\underset{\sim}{T}{\underset{m}{i}}_{i}^{G}\right] \text { (for } i<2\right) \text { we have } \\
b_{0}\left\lceil N \neq c_{0}\left\lceilN \text { and } \left\{b _ { 0 } \left\lceil N, c_{0}\lceil N\} \cap\left\{b _ { 1 } \left\lceilN, c_{1}\lceil N\}=\emptyset\right.\right.\right.\right.\right.\right. \\
\text { and } \left.f+g=b_{0}+c_{0}=b_{1}+c_{1}\right\}
\end{array}
$$

By our assumption on $P$ we know that

$(\otimes)_{1}$ for each distinct $x, y \in P$ there are $\ell_{0}, m_{0}, \ell_{1}, m_{1}, N<\omega$ such that $(x, y) \in$ $A_{\ell_{0}, m_{0}, \ell_{1}, m_{1}}^{N}$.

Since the sets $A_{\ell_{0}, m_{0}, \ell_{1}, m_{1}}^{N}$ are $\Sigma_{1}^{1}$, we may use Lemma 2.4 to choose a perfect set $P^{*} \subseteq P$ and an increasing sequence $0=n_{0}<n_{1}<n_{2}<n_{3}<\ldots$ such that

$(\otimes)_{2}$ for each $k<\omega$ and any distinct $x, y \in P^{*}$ we have:

(a) if $x\left\lceil n_{k+1} \neq y\left\lceil n_{k+1}, \ell_{0}, m_{0}, \ell_{1}, m_{1}, N \leq k\right.\right.$, then for all $x^{\prime}, y^{\prime} \in P^{*}$ satisfying $x\left\lceil n_{k+1}=x^{\prime}\left\lceil n_{k+1}\right.\right.$ and $y\left\lceil n_{k+1}=y^{\prime}\left\lceil n_{k+1}\right.\right.$ we have

$$
(x, y) \in A_{\ell_{0}, m_{0}, \ell_{1}, m_{1}}^{N} \Leftrightarrow\left(x^{\prime}, y^{\prime}\right) \in A_{\ell_{0}, m_{0}, \ell_{1}, m_{1}}^{N},
$$

(b) the set $\left\{z\left\lceil n_{k+1}: z \in P^{*} \wedge z\left\lceil n_{k}=x\left\lceil n_{k}\right\}\right.\right.\right.$ has exactly two elements.

Now, by induction on $j \leq 21$ we may choose $0=k_{0}<k_{1}<k_{2}<\ldots<k_{j}<\ldots<$ $k_{20}<k_{21}$ and $A \subseteq P^{*}$ such that

$(\otimes)_{3}|A|=2^{20}$, say $A=\left\{x_{0}, \ldots, x_{2^{20}-1}\right\}$,

$(\otimes)_{4}$ if $j \leq 20, x, y \in A$ and $x\left\lceil n_{k_{j}} \neq y\left\lceil n_{k_{j}}\right.\right.$, then $(x, y) \in A_{\ell_{0}, m_{0}, \ell_{1}, m_{1}}^{N}$ for some $\ell_{0}, m_{0}, \ell_{1}, m_{1}, N<k_{j}$ 
$(\nabla)_{5}$ if $j<20$ and $x \in A$, then there is $y \in A$ such that $x\left\lceil n_{k_{j}}=y\left\lceil n_{k_{j}}\right.\right.$ but $x\left\lceil n_{k_{j+1}} \neq y\left\lceil n_{k_{j+1}}\right.\right.$.

Let $\underset{\sim}{P^{*}}, \underset{\sim}{\bar{n}}, \underset{\sim}{A}=\left\{{\underset{\sim}{x}}_{0}, \ldots,{\underset{\sim}{2^{20}-1}}^{A}\right\}$, and $\underset{\sim}{\bar{k}}$ be $\mathbb{P}$-names for the objects appearing in $(\nabla)_{1}-(\nabla)_{5}$ and let a condition $p \in G$ force that they have the properties listed there.

Passing to a stronger condition we may also demand that

$(\bigotimes)_{6} p$ decides the values of $\underset{\sim}{k_{0}}, \underset{\sim}{k_{1}}, \ldots,{\underset{\sim}{21}}_{21}$, say $p \Vdash \underset{\sim}{k_{j}}=k_{j}$ for $j \leq 21$,

$(\otimes)_{7} p$ decides the values of $\sim_{\sim}, \sim_{\sim}, \ldots, n_{k_{21}}$, say $p \Vdash n_{i}=n_{i}$ for $i \leq k_{21}$,

$(\otimes)_{8} p$ decides the values of $\underset{\sim}{x}\left\lceil\left\lceil n_{k_{21}}, \ldots,{\underset{\sim}{2} 2^{20}-1}\left\lceil n_{k_{21}}\right.\right.\right.$, say $p \Vdash x_{i}\left\lceil n_{k_{21}}=\sigma_{i}^{*}\right.$ for $i<2^{20}$,

$(\otimes)_{9} n^{p}>n_{k_{21}}$ and $m_{*}^{p}>k_{21}$.

Note that it follows from $(\otimes)_{3}+(\otimes)_{5}$ that

$(\otimes)_{10}$ if $i<j<2^{20}$, then $\sigma_{i}^{*} \neq \sigma_{j}^{*}$.

Since $p$ forces that $x_{i}$ 's have the properties listed in $(\otimes)_{1}$ and $(\otimes)_{3}-(\otimes)_{5}$, there are $\sigma_{i} \in n^{p} 2\left(\right.$ for $\left.i<2^{20}\right)$ such that

$(\otimes)_{11} \sigma_{i}^{*} \triangleleft \sigma_{i}$ for each $i<2^{20}$, and

$(\otimes)_{12}$ if $i, j<2^{20}$ are distinct, then for some $\ell_{0}(i, j), m_{0}(i, j), \ell_{1}(i, j), m_{1}(i, j)<$ $m_{*}^{p}$ and $b_{0}(i, j) \in t_{\ell_{0}(i, j)}^{p} \cap^{n^{p}} 2, c_{0}(i, j) \in t_{m_{0}(i, j)}^{p} \cap^{n^{p}} 2, b_{1}(i, j) \in t_{\ell_{1}(i, j)}^{p} \cap^{n^{p}} 2$, $c_{1}(i, j) \in t_{m_{1}(i, j)}^{p} \cap n^{p} 2$ we have

(a) $\sigma_{i}+\sigma_{j}=b_{0}(i, j)+c_{0}(i, j)=b_{1}(i, j)+c_{1}(i, j)$, and

(b) $\left\{b_{0}(i, j), c_{0}(i, j)\right\} \cap\left\{b_{1}(i, j), c_{1}(i, j)\right\}=\emptyset$,

$(\otimes)_{13}$ if $i, i^{\prime}, j, j^{\prime}<2^{20}$ and $k<n^{p}$ are such that $\sigma_{i}\left\lceil k=\sigma_{i^{\prime}}\left\lceil k \neq \sigma_{j}\left\lceil k=\sigma_{j^{\prime}}\lceil k\right.\right.\right.$, then

$$
\left\langle\ell_{0}(i, j), m_{0}(i, j), \ell_{1}(i, j), m_{1}(i, j)\right\rangle=\left\langle\ell_{0}\left(i^{\prime}, j^{\prime}\right), m_{0}\left(i^{\prime}, j^{\prime}\right), \ell_{1}\left(i^{\prime}, j^{\prime}\right), m_{1}\left(i^{\prime}, j^{\prime}\right)\right\rangle .
$$

It follows from $(\otimes)_{10^{-}}(\otimes)_{12}$ that there are no repetitions in the list $b_{0}(i, j), c_{0}(i, j)$, $b_{1}(i, j), c_{1}(i, j)$.

By Claim 3.1.2 (2), for distinct $i, j<2^{20}$ we can find $\ell<2$ and distinct $\alpha, \beta$ from $u^{p}$ such that

- either $\left\{b_{\ell}(i, j), c_{\ell}(i, j)\right\}=\left\{\eta_{\alpha}^{p}, \eta_{\beta}^{p}\right\}$ (in which case we set $h(i, j)=1$ ),

- or $\left\{b_{\ell}(i, j), c_{\ell}(i, j)\right\}=\left\{\eta_{\alpha}^{p}+\rho_{\alpha, \beta}^{p}, \eta_{\beta}^{p}\right\}$ (and then we set $h(i, j)=0$ ),

- or $\left\{b_{\ell}(i, j), c_{\ell}(i, j)\right\}=\left\{\eta_{\alpha}^{p}+\rho_{\alpha, \beta}^{p}, \eta_{\alpha}^{p}\right\}$ (and then we also set $h(i, j)=0$ ).

Note that

$(\otimes)_{14}$ if $i, j, k<2^{20}$ are pairwise distinct, then $h(i, j)=1$, or $h(j, k)=1$, or $h(i, k)=1$.

Why? First suppose that for some $\alpha<\beta, \gamma<\delta$ and $\varepsilon<\zeta$ from $u^{p}$ we have

$$
\begin{aligned}
& \sigma_{i}+\sigma_{j}=b_{0}(i, j)+c_{0}(i, j)=\eta_{\alpha}^{p}+\eta_{\beta}^{p}+\rho_{\alpha, \beta}^{p}, \\
& \sigma_{j}+\sigma_{k}=b_{0}(j, k)+c_{0}(j, k)=\eta_{\gamma}^{p}+\eta_{\delta}^{p}+\rho_{\gamma, \delta}^{p}, \\
& \sigma_{i}+\sigma_{k}=b_{0}(i, k)+c_{0}(i, k)=\eta_{\varepsilon}^{p}+\eta_{\zeta}^{p}+\rho_{\varepsilon, \zeta}^{p} .
\end{aligned}
$$

Then

$$
0=\left(\eta_{\alpha}^{p}+\eta_{\beta}^{p}+\rho_{\alpha, \beta}^{p}\right)+\left(\eta_{\gamma}^{p}+\eta_{\delta}^{p}+\rho_{\gamma, \delta}^{p}\right)+\left(\eta_{\varepsilon}^{p}+\eta_{\zeta}^{p}+\rho_{\varepsilon, \zeta}^{p}\right) .
$$

However, by the linear independence, this is not possible (the $\rho$ 's cannot be cancelled). 
Second, suppose

$$
\begin{aligned}
& \sigma_{i}+\sigma_{j}=b_{0}(i, j)+c_{0}(i, j)=\eta_{\alpha}^{p}+\eta_{\alpha}^{p}+\rho_{\alpha, \beta}^{p}=\rho_{\alpha, \beta}^{p}, \\
& \sigma_{j}+\sigma_{k}=b_{0}(j, k)+c_{0}(j, k)=\eta_{\gamma}^{p}+\eta_{\delta}^{p}+\rho_{\gamma, \delta}^{p}, \\
& \sigma_{i}+\sigma_{k}=b_{0}(i, k)+c_{0}(i, k)=\eta_{\varepsilon}^{p}+\eta_{\zeta}^{p}+\rho_{\varepsilon, \zeta}^{p} .
\end{aligned}
$$

Then $\rho_{\alpha, \beta}^{p}=\left(\eta_{\gamma}^{p}+\eta_{\delta}^{p}+\rho_{\gamma, \delta}^{p}\right)+\left(\eta_{\varepsilon}^{p}+\eta_{\zeta}^{p}+\rho_{\varepsilon, \zeta}^{p}\right)$, and this is again not possible by the linear independence.

Thirdly, the assumption that $\sigma_{i}+\sigma_{j}=\rho_{\alpha, \beta}^{p}, \sigma_{j}+\sigma_{k}=\rho_{\gamma, \delta}^{p}$ and $\sigma_{i}+\sigma_{k}=$ $\eta_{\varepsilon}^{p}+\eta_{\zeta}^{p}+\rho_{\varepsilon, \zeta}^{p}$ leads to

again a clear contradiction.

$$
\rho_{\alpha, \beta}^{p}+\rho_{\gamma, \delta}^{p}=\eta_{\varepsilon}^{p}+\eta_{\zeta}^{p}+\rho_{\varepsilon, \zeta}^{p},
$$

Finally, the configuration $\sigma_{i}+\sigma_{j}=\rho_{\alpha, \beta}^{p}, \sigma_{j}+\sigma_{k}=\rho_{\gamma, \delta}^{p}$ and $\sigma_{i}+\sigma_{k}=\rho_{\varepsilon, \zeta}^{p}$ is also impossible.

Using Lemma 2.2 we may find $A \subseteq\left\{i: i<2^{20}\right\}$ such that

$(\otimes)_{15}$ (a) $|A| \geq 5$, and $\mathcal{A}=\left\{\sigma_{i}: i \in A\right\}$ contains a 4-arrangement (see Definition 2.11), and

(b) $A$ is $h$-homogeneous in color 1, i.e., $h(i, j)=1$ for $i<j$ from $A$ (remember $\left.(\otimes)_{3}+(\otimes)_{5}\right)$. Now, $(\otimes)_{15}(\mathrm{~b})$ implies that

$$
\mathcal{A}+\mathcal{A} \subseteq\left\{\eta_{\alpha}^{p}: \alpha \in u^{p}\right\}+\left\{\eta_{\alpha}^{p}: \alpha \in u^{p}\right\} .
$$

Hence, by Lemma 2.3(b), there is $x \in{ }^{n^{p}} 2$ such that $\mathcal{A}+x \subseteq\left\{\eta_{\alpha}^{p}: \alpha \in u^{p}\right\}$. Since $\mathcal{A}+x$ contains a 4 -arrangement we may find $\alpha<\beta<\gamma$ such that $\eta_{\alpha}^{p}, \eta_{\beta}^{p}, \eta_{\gamma}^{p} \in \mathcal{A}+x$ and

$$
\begin{aligned}
& \min \left\{k<n^{p}: \eta_{\alpha}^{p}(k) \neq \eta_{\gamma}^{p}(k)\right\}=\min \left\{k<n^{p}: \eta_{\beta}^{p}(k) \neq \eta_{\gamma}^{p}(k)\right\} \\
&<\min \left\{k<n^{p}: \eta_{\alpha}^{p}(k) \neq \eta_{\beta}^{p}(k)\right\} .
\end{aligned}
$$

Now, $\left(\eta_{\alpha}^{p}+x\right),\left(\eta_{\beta}^{p}+x\right),\left(\eta_{\gamma}^{p}+x\right) \in \mathcal{A}$ so let $i, j, k<2^{20}$ be such that $\eta_{\alpha}^{p}+x=$ $\sigma_{i}, \eta_{\beta}^{p}+x=\sigma_{j}$ and $\eta_{\gamma}^{p}+x=\sigma_{k}$. Then

$$
\eta_{\alpha}^{p}+\eta_{\gamma}^{p}=\sigma_{i}+\sigma_{k}=b_{0}(i, k)+c_{0}(i, k)=b_{1}(i, k)+c_{1}(i, k) .
$$

By $(\otimes)_{15}(\mathrm{~b})$ we know that $h(i, k)=1$, so for some $x<2$ there are distinct $\varepsilon, \zeta \in u^{p}$ such that $\left\{=b_{x}(i, k), c_{x}(i, k)\right\}=\left\{\eta_{\varepsilon}^{p}, \eta_{\zeta}^{p}\right\}$. Then $\eta_{\alpha}^{p}+\eta_{\gamma}^{p}=\eta_{\varepsilon}^{p}+\eta_{\zeta}^{p}$ and by the linear independence of $\left\langle\eta_{\delta}^{p}: \delta \in u\right\rangle\left\langle\left\langle\rho_{\delta, \xi}^{p}: \delta<\xi \wedge \delta, \xi \in u\right\rangle\right.$ necessarily $\left\{\eta_{\varepsilon}^{p}, \eta_{\zeta}^{p}\right\}=\left\{\eta_{\alpha}^{p}, \eta_{\gamma}^{p}\right\}$. But now Claim $3.1 .2(1)$ gives that $\left\{b_{1-x}(i, k), c_{1-x}(i, k)\right\}=\left\{\eta_{\alpha}^{p}+\rho_{\alpha, \gamma}^{p}, \eta_{\beta}^{p}+\rho_{\alpha, \gamma}^{p}\right\}$. Consequently, using $(\otimes)_{12}+(7)$ we get

$$
\left\{K_{\alpha}^{p}, K_{\gamma}^{p}\right\}=\left\{\ell_{x}(i, k), m_{x}(i, k)\right\} \quad \text { and } \quad\left\{\ell_{\alpha, \gamma}^{p}\right\}=\left\{\ell_{1-x}(i, k), m_{1-x}(i, k)\right\} .
$$

Therefore,

$$
\left\{K_{\alpha}^{p}, K_{\gamma}^{p}, \ell_{\alpha, \gamma}^{p}\right\}=\left\{\ell_{0}(i, k), m_{0}(i, k), \ell_{1}(i, k), m_{1}(i, k)\right\} .
$$

Similarly, considering $(j, k, \beta, \gamma)$ instead of $(i, k, \alpha, \gamma)$, we show that

$$
\left\{K_{\beta}^{p}, K_{\gamma}^{p}, \ell_{\beta, \gamma}^{p}\right\}=\left\{\ell_{0}(j, k), m_{0}(j, k), \ell_{1}(j, k), m_{1}(j, k)\right\} .
$$

By $(\otimes)_{13}$ we have

$$
\left\{\ell_{0}(i, k), m_{0}(i, k), \ell_{1}(i, k), m_{1}(i, k)\right\}=\left\{\ell_{0}(j, k), m_{0}(j, k), \ell_{1}(j, k), m_{1}(j, k)\right\}
$$

and this implies that $\left\{K_{\alpha}^{p}, K_{\gamma}^{p}, \ell_{\alpha, \gamma}^{p}\right\}=\left\{K_{\beta}^{p}, K_{\gamma}^{p}, \ell_{\beta, \gamma}^{p}\right\}$, contradicting clause (6) of the definition of $\mathbb{P}$. 
Theorem 3.2. (1) Assume that a $\Sigma_{2}^{0}$ set $B \subseteq \omega_{2}$ satisfies (A) of Theorem 3.1 . Let $\mathbb{Q}$ be a ccc forcing notion. Then $\Vdash_{\mathbb{Q}}$ "B satisfies $(\boldsymbol{\phi})$ ".

(2) Suppose $\kappa$ is an infinite cardinal satisfying $\kappa^{\omega_{0}}=\kappa$. Then for some ccc forcing notion $\mathbb{Q}_{0}$ we have

$$
\Vdash_{\mathbb{Q}_{0}} " \mathfrak{c}=\kappa \text { and there is a } \Sigma_{2}^{0} \text { set } B \subseteq{ }^{\omega} 2 \text { satisfying }(\mathbf{(}) " .
$$

(3) Assume $\mathbf{C H}$. Let $\mathbb{C}_{\omega_{2}}$ be the forcing notion adding $\omega_{2}$ Cohen reals. Then in $\mathbf{V}^{\mathbb{C}_{\omega_{2}}}$ the following holds:

If $B \subseteq{ }^{\omega} 2$ is Borel , $\left\langle\eta_{\alpha}: \alpha<\omega_{2}\right\rangle \subseteq{ }^{\omega} 2$ and

$$
\left(\forall \alpha<\beta<\omega_{2}\right)\left(\left|\left(B+\eta_{\alpha}\right) \cap\left(B+\eta_{\beta}\right)\right| \geq 4\right),
$$

then there is a perfect set $P \subseteq{ }^{\omega} 2$ such that

$$
(\forall x, y \in P)(|(B+x) \cap(B+y)| \geq 4) .
$$

(4) Assume $\mathbf{C H}$. Then for some ccc forcing notion $\mathbb{Q}_{1}$ we have $\Vdash_{\mathbb{Q}}$ "c $=\omega_{2}$ and there is a $\Sigma_{2}^{0}$ set $B \subseteq \omega_{2}$ satisfying

$(\circledast)_{1}$ for some sequence $\left\langle h_{\alpha}: \alpha<\omega_{1}\right\rangle$ of pairwise distinct elements of ${ }^{\omega} 2$ we have $\left|\left(h_{\alpha}+B\right) \cap\left(h_{\beta}+B\right)\right| \geq 4$ for all $\alpha, \beta<\omega_{1}$, but

$(\circledast)_{2}$ there is no sequence $\left\langle g_{\alpha}: \alpha<\omega_{2}\right\rangle$ of pairwise distinct elements of ${ }^{\omega_{2}}$ such that $\left|\left(g_{\alpha}+B\right) \cap\left(g_{\beta}+B\right)\right| \geq 4$ for all $\alpha, \beta<\omega_{2}$ ".

Proof. (1) For a $\Sigma_{2}^{0}$ set $B \subseteq{ }^{\omega} 2$, the set

$$
\mathcal{Z}_{B}=\left\{(x, y) \in{ }^{\omega} 2 \times{ }^{\omega} 2:|(B+x) \cap(B+y)|>2\right\}
$$

is $\Sigma_{2}^{0}$. One can easily see this by noting that $(x, y) \in \mathcal{Z}_{B}$ if and only if

$$
\left(\exists t_{0}, t_{1}, t_{2} \in B\right)\left(x+y+t_{0} \in B \wedge x+y+t_{1} \in B \wedge x+y+t_{2} \in B \wedge\left|\left\{t_{0}, t_{1}, t_{2}\right\}\right|=3\right)
$$

and remembering that continuous images of $\Sigma_{2}^{0}$ subsets of ${ }^{5}\left({ }^{\omega} 2\right)$ are $\Sigma_{2}^{0}$. Consequently, for a $\Sigma_{2}^{0}$ set $B \subseteq{ }^{\omega_{2}}$, the formula "there is a perfect set $P \subseteq{ }^{\omega_{2}}$ such that $P \times P \subseteq \mathcal{Z}_{B}$ " is $\Sigma_{2}^{1}$, and thus absolute for forcing extensions.

Since $A \times A \subseteq \mathcal{Z}_{B}$ if and only if $|(B+x) \cap(B+y)|>2$ for $x, y \in A$, the assertion should be clear.

(2) Let $\mathbb{P}$ be the forcing notion given by Theorem 3.1 and let $\mathbb{C}_{\kappa}$ be the forcing notion adding $\kappa$ Cohen reals (with finite conditions). It follows from (1) above (and Theorem 3.1) that the composition $\mathbb{Q}_{0}=\mathbb{P} * \mathbb{C}_{\kappa}$ has the required property.

(3) First note that for a Borel set $B \subseteq \omega^{\omega} 2$, the set $\mathcal{Z}_{B}=\left\{(x, y) \in{ }^{\omega}{ }_{2} \times{ }^{\omega_{2}} 2\right.$ : $|(B+x) \cap(B+y)|>2\}$ is $\Sigma_{1}^{1}$. Now, by Shelah [5, Fact 1.16], after adding $\omega_{2}$ Cohen reals over a model of $\mathbf{C H}$, every analytic subset of ${ }^{\omega} 2 \times{ }^{\omega}{ }_{2}$ (so sets $\mathcal{Z}_{B}$ in particular) which contains a square of side length $\omega_{2}$ contains a perfect square.

See more in Rosłanowski and Shelah [4, Section 3].

(4) Let $\mathbb{P}$ be the forcing notion given by Theorem 3.1, let $\mathbb{C}_{\omega_{2}}$ be the forcing notion adding $\omega_{2}$ Cohen reals, and let $\mathbb{Q}_{1}=\mathbb{P} * \mathbb{C}_{\omega_{2}}$. In $\mathbf{V}^{\mathbb{Q}_{1}}$ consider the $\Sigma_{2}^{0}$ set $B$ added by $\mathbb{P}$. As in $(2)$ above, $\Vdash_{\mathbb{Q}_{1}}$ " $B$ satisfies $(\boldsymbol{A})$ ", so in particular $\vdash_{\mathbb{Q}_{1}}$ " $B$ satisfies $(\circledast)_{1}$ ". Since $\mathbf{V}^{\mathbb{P}} \models \mathbf{C H}$, we may also use (3) to argue that $\Vdash_{\mathbb{Q}_{1}}$ " $B$ satisfies $(\circledast)_{2} "$.

Theorem 3.3. Assume $\mathbf{M A}+\neg \mathbf{C H}$. Then there is a $\Sigma_{2}^{0}$ set $B \subseteq \omega_{2}$ satisfying (\$) of Theorem 3.1 , 
Proof. Standard consequence of the proof of Theorem 3.1. With notation as there, let $\mathcal{M}$ be a transitive model of a large part of ZFC and such that $\omega_{1}^{\mathcal{M}}=\omega_{1}$ and $|\mathcal{M}|=\omega_{1}$. (For instance, take the transitive collapse of $\mathcal{N} \prec \mathcal{H}(\chi),|\mathcal{N}|=\omega_{1}$, $\omega_{1} \subseteq \mathcal{N}$.) Then $\mathbb{P} \in \mathcal{M}$. By $\mathbf{M A}+\neg \mathbf{C H}$ we may find an $\mathcal{M}$-generic $G \subseteq \mathbb{P}$. Consider $\mathcal{M}[G]$ which is a model of a large part of ZFC. By the proof of Theorem 3.1 there are $\left\langle T_{m}: m<\omega\right\rangle$ and $\left\langle\eta_{\alpha}: \alpha<\omega_{1}\right\rangle$ belonging to $\mathcal{M}[G]$ such that

$(\boldsymbol{A})^{\mathcal{M}[G]}$ in $\mathcal{M}[G]$

(a) $T_{m} \subseteq \omega>2$ is a tree with no terminal nodes (for every $m<\omega$ ) and $\eta_{\alpha} \in{ }^{\omega_{2}}$ (for each $\left.\alpha<\omega_{1}\right)$,

(b) $\left|\left(\eta_{\alpha}+\bigcup_{m<\omega}\left[T_{m}\right]\right) \cap\left(h_{\beta}+\bigcup_{m<\omega}\left[T_{m}\right]\right)\right| \geq 4$,

(c) for every perfect set $P \subseteq \omega_{2}$ there are $f, g \in P$ with $\mid\left(f+\bigcup_{m<\omega}\left[T_{m}\right]\right) \cap$ $\left(g+\bigcup_{m<\omega}\left[T_{m}\right]\right) \mid \leq 2$.

The properties stated in $(\boldsymbol{A})^{\mathcal{M}[G]}(\mathrm{a}),(\mathrm{b})$ are clearly absolute between $\mathcal{M}$ and $\mathbf{V}$. By an argument as in Theorem $\underline{3.2}(1)$, the property stated in $(\boldsymbol{A})^{\mathcal{M}[G]}$ (c) is $\Pi_{2}^{1}$, so also absolute between $\mathcal{M}$ and $\mathbf{V}$.

For the completeness of the picture we would like to know the answer to the following problem (compare this with Theorem $\underline{3.2}(3)$ ).

Problem 3.4. Is it consistent that for every Borel set $B \subseteq{ }^{\omega_{2}}$,

if there is a sequence $\left\langle\eta_{\alpha}: \alpha<\omega_{1}\right\rangle$ of distinct elements of $\omega_{2}$ such that

$$
\left(\forall \alpha<\beta<\omega_{1}\right)\left(\left|\left(B+\eta_{\alpha}\right) \cap\left(B+\eta_{\beta}\right)\right| \geq 4\right)
$$

then there is a perfect set $P \subseteq{ }^{\omega}{ }^{2}$ such that

$$
(\forall x, y \in P)(|(B+x) \cap(B+y)| \geq 4) ?
$$

\section{ACKNOWLEDGMENT}

We would like to thank the anonymous referee for very valuable comments and corrections.

\section{REFERENCES}

[1] Marek Balcerzak, Andrzej Rosłanowski, and Saharon Shelah, Ideals without ccc, J. Symbolic Logic 63 (1998), no. 1, 128-148, DOI 10.2307/2586592. MR 1610790

[2] Thomas Jech, Set theory, Springer Monographs in Mathematics, Springer-Verlag, Berlin, 2003. The third millennium edition, revised and expanded. MR.1940513

[3] Jan Mycielski, Independent sets in topological algebras, Fund. Math. 55 (1964), 139-147, DOI 10.4064/fm-55-2-139-147. MR0173645

[4] Andrzej Roslanowski and Saharon Shelah, Borel sets without perfectly many overlapping translations. Reports on Mathematical Logic, submitted, arxiv:1806.06283.

[5] Saharon Shelah, Borel sets with large squares, Fund. Math. 159 (1999), no. 1, 1-50. MR 1669643

Department of Mathematics, University of Nebraska at Omaha, Omaha, Nebraska 68182-0243

Email address: aroslanowski@unomaha.edu

Department of Mathematics, University of Nebraska at Omaha, Omaha, Nebraska 68182-0243

Email address: vrykov@unomaha.edu 